\title{
Reprodutibilidade e Validade da Avaliação Doplervelocimétrica no Diagnóstico de Massas Ovarianas
}

\section{Reproducibility and validity of Doppler evaluation of ovarian masses}

Aluna: Ana Carolina da Silva Marchesini

Orientador: Prof. Dr. Francisco José Candido dos Reis

Dissertação de Mestrado apresentada o Departamento de Ginecologia e Obstetrícia da Faculdade de Medicina de Ribeirão Preto - Universidade de São Paulo, em 19 de janeiro de 2006.

Introdução: a ultra-sonografia é o exame menos invasivo capaz de detectar alterações de pequeno volume na região anexial e o estudo Doppler colorido permite uma avaliação indireta do metabolismo da lesão. No entanto, as neoplasias malignas podem apresentar áreas de tecido ovariano benigno e dificultar o diagnóstico diferencial ultra-sonográfico. Objetivos: este estudo objetivou avaliar a validade e a reprodutibilidade do estudo Doppler no diagnóstico diferencial das massas anexiais; e verificar se algum parâmetro específico da doplervelocimetria (PVS, EDV, IP ou IR) teria maior eficácia diagnóstica. Métodos: foi realizado estudo tipo observacional prospectivo incluindo 15 (21,12\%) pacientes com diagnóstico de neoplasia maligna e 56 (78,88\%) com diagnóstico de doença benigna do ovário. Para o estudo Doppler as massas eram setorizadas em quatro quadrantes a partir dos cortes longitudinal e transverso. Todos os quadrantes foram insonados individualmente para amostragem dopplervelocimétrica. Resultados: na avaliação dos quadrantes apenas $46,47 \%$ das massas (33 lesões) apresentaram vascularização arterial nos quatro quadrantes. O coeficiente de variação (CV) para os diversos parâmetros do Doppler variou entre os seguintes valores: para o IR de 11,7 a $41,7 \%$, para o
IP de 21,1 a $32,9 \%$, para o PVS de 35,0 a $55,4 \%$, e para o VDF de 40,1 a 50,2\%. A ultra-sonografia morfológica apresentou sensibilidade de $86,7 \%$, especificidade de 53,6\%, VPP de 33,3\% e VPN de 93,75\%; com área sob a curva igual a 0,69 (IC 95\%: 0,57 e 0,81, p = 0,0009). Quando se associou a dopplervelocimetria aos achados morfológicos, a sensibilidade do método foi de 93,3\%, especificidade de 62,5\%, VPP de 40\% e VPN de 97,22\%; com área sob a curva igual a 0,80 (IC 95\% 0,70 e 0,90, p < 0,0001). Comparando o método morfológico com o mesmo método acrescido do estudo Doppler, observou-se melhor desempenho do exame utilizando os dois métodos com diferença estatística significativa $(p=0,0003)$. Conclusões: os resultados permitem concluir que o indice de resistência foi o parâmetro dopplervelocimétrico com menor variabilidade, portanto o mais reprodutivel. Apenas uma medida dopplervelocimétrica da massa ovariana não foi representativa das características vasculares da lesão. O estudo Doppler melhorou os parâmetros de sensibilidade e especificidade do exame ultra-sonográfico no estudo de massas ovarianas.

PALAVRAS-CHAVE: Câncer de ovário; Massa anexial; Ultra-sonografia; Doppler

Resumo de Tese

\section{Avaliação da resposta à quimioterapia primária de pacientes com câncer de mama estadio II e III submetidas ao tratamento com FEC50 e AC}

\section{Evaluations of the locoregional response to primary chemotherapy in patients with breast cancer at stages II and III that received FEC50 and AC}

Autor: Eduardo Carvalho Pessoa

Orientador: Prof. Dr. Gilberto Uemura

Dissertação apresentada ao Programa de Pós-Graduação em Ginecologia, Obstetrícia e Mastologia, Faculdade de Medicina de Botucatu - UNESP, para obtenção do Título de Mestre, em 16 de setembro de 2005.

Objetivo: avaliar a resposta loco-regional a quimioterapia primária nas pacientes com câncer de mama estadio II e III. Sujeitos e Métodos: foi realizado um estudo clínico retrospectivo e analítico de 97 pacientes no estadio II e III do CAM "Prof. Laurival Antonio De Luca" - Faculdade de Medicina de Botucatu - UNESP, no período de janeiro de 1993 a dezembro de 2004, submetidas a 3 ou 4 ciclos de quimioterapia primária com FEC50 (5-fluorouracil - 500mg/m², Epirrubicina - 50mg/ $\mathrm{m}^{2}$ e ciclofosfamida $-500 \mathrm{mg} / \mathrm{m}^{2}$ ) ou AC (doxorrubicina - $50 \mathrm{mg} / \mathrm{m}^{2}$ e ciclofosfamida $-500 \mathrm{mg} / \mathrm{m}^{2}$ ) e posterior- mente ao tratamento loco-regional cirúrgico conservador ou radical. Para estudo da associação entre as variáveis foram utilizados os testes de qui-quadrado e o Exato de Fisher. Para as variáveis quantitativas foi utilizado o coeficiente de correlação de Pearson. Resultados: a média de idade da população estuda foi de 52,2 anos. No estádio II tivemos $56,8 \%$ dos casos e no estádio III 43,2\%. Aproximadamente $50 \%$ das pacientes receberam FEC50 e 50\% AC. Obtivemos uma resposta clinica objetiva com o tratamento quimioterápico primário em $64,9 \%$ dos casos. A resposta clinica com- 